\title{
Modelling of the bread recipe composition to improve effectiveness of the production process
}

\author{
Elena Ponomareva*, Svetlana Lukina, Nadezhda Alekhina, Tatiana Malyutina, and \\ Svetlana Pavlovskaya
}

Voronezh State University of Engineering Technologies, Faculty of Technology, Revolution Avenue, 19, Voronezh, Russia

\begin{abstract}
This paper looks into the combined influence of untraditional types of raw materials on the quality of wheat flour bread and the effectiveness of the technological process. To estimate that, the methods of mathematical planning and experiment optimisation were applied. The main factors were the dosage of recipe components such as turmeric and liquid grape sugar, the output parameters were unit volume and shape stability of a product. The optimisation of the recipe composition was carried out by the method of folding partial criteria in generalised additive criteria. Rational criteriameanings were determined: the dosage of liquid grape sugar $-2,50 \%$, turmeric $-2,75 \%$, which guarantee the production of products with the best combination of values of specific volume and dimensional stability. Basing on the data obtained, there was elaborated the formula of the bread «Merita» (TU 9110-515-02068108-2019) which is characterised with improved quality and higher nutritional value. The application of this technology will allow to increase output of product by $3 \%$ and reduce the production cycle by 1,5 hours.
\end{abstract}

\section{Introduction}

The primary task of the alimentary industry is to increase the manufacturing of enriched, functional, medical-prophylactic and specialised high-quality products which is reflected in the documents ratified on the governmental level in the sphere of health nutrition of the Russian population.

The modern direction in the development of the bakery branch of industry is a process of looking for new types of raw materials sources with rich composition of biologically active substances, which are vital for a human body to provide a correct functioning of all systems and treating a number of diseases. In order to achieve a sustainably high quality of bakery products and to increase the effectiveness of production process it is necessary to combine purposefully the receipt composition using modern computer technologies and methods of modelling and experiment optimisation $[1,2]$.

\footnotetext{
*Corresponding author: elena6815@ya.ru
} 
The technology-scientific literature review has shown the topicality and expediency of the implementation of untraditional types of raw materials in order to enrich bakery products, to giving them medical and prophylactic qualities. The choice of used in the study recipe components is attributable to their chemical composition and functional qualities.

Turmeric is a perspective enriching agent of vegetable origin. In the alimentary industry it is applied in the form of powder made of dried roots of the plant Curcuma longa as a spice and natural colourant (E 100). The chemical composition of turmeric is characterised by high content of dietary fibers ( $21 \mathrm{~g}, 70 \%$ of daily needs), iron (3 times higher than daily needs), vitamin B6 (1,8 mg, $90 \%$ of daily needs), ascorbic acid (25,9 mg, $43 \%$ of daily needs), potassium ( $2525 \mathrm{mg}, 72 \%$ of daily needs) which are necessary for strengthening the immunity, body detoxication from heavy metals and toxic substances, providing correct functioning of circulatory, nervous, muscular and cardiovascular systems. The positive effect is also provided by the content of such components as essential oils and their elements (sabinene, borneol, zingiberene, terpen alcohol, phellandren). The active substance of turmeric curcumin has anti-inflammatory, autoimmune, anticarcinogenic, antimutagenic and antioxidant effects $[4,5]$.

In order to improve nutritional value and reduce sugar content of a product it is effective to use liquid grape sugar produced by the rectification of grape must. The carbohydrate composition of liquid grape sugar contains mixture of glucose $(44 \%)$ and fructose $(33 \%)$ which is a good alternative to white sugar which mostly consists of pure sucrose. Moreover, among valuable elements there are vitamins of groups B, C, H, PP, micro- and macro elements. One of the most valuable bioactive elements is a natural antioxidant flavonoid quercetin which has antiallergenic affect.

The objective of the study is to define the rational dosage of components of a bakery product.

\section{Material and methods}

Raw material used in the study: wheat flower of first grade (GOST 56574-2017), pressed bakery yeast (GOST R 54731-2011), edible salt «Valetek» (TU 9192-050-17028327), liquid grape sugar (GOST 33222-2015), sunflower oil (GOST 1129-2013), turmeric (GOST ISO 5562-2017), drinking water (SanPiN 2.1.4.1074-01).

Objects of the study: bread samples baked basing on the recipe of «Saratovskij kalach» (GOST R 58233-2018) with the use of untraditional raw materials.

Samples of bakery products were cooked while maintaining following parameters: straight dough method with humidity of $46,0 \%$ and fermentation at a temperature of $30 \pm 2{ }^{\circ} \mathrm{C}$ during 90 minutes, proofing of test workpieces at $38 \pm 2{ }^{\circ} \mathrm{C}$ and relative air humidity maintained at $80-85 \%$ during 20 minutes. The bread quality indicators were indicated according to the methods mentioned in the handbook [6].

In order to determine rational recipe composition of bread there were chosen the dosages of components as input parameters in percentage $(\%)$ by flour mass: $x_{1}-$ liquid grape sugar, $x_{2}$ - turmeric. The process of intervals modification depended on technological characteristics and bakery products quality based on preliminary studies. Unit volume $\left(y_{1}\right.$, $\mathrm{sm}^{3} / 100 \mathrm{~g}$ ) and shape stability ( $y_{2}$, conv. units) were chosen as output parameters [7].

\section{Results and discussion}

The study was carried out under laboratory conditions. Central composite rotatable design was applied during the experiment. The data processing was carried out according to statistic criteria by Cochrane, Student and Fisher with confidence level of 0,95 . The 
optimisation of dough recipe with the use of untraditional raw materials was carried out with the application of mathematical statistics and differential calculus.

The testing was conducted using full factorial experiment type $2^{3}$, double replication according to planning matrix consisting of 5 parallel experiments in the centre of the plan and experiments in «star» points with the length the «star shoulder» $\pm 1,41$ [9].

The results obtained after the statistical processing were represented as regression equations which accurately describe the dependance of bread quality indicators on recipe components dosage:

$$
\begin{gathered}
y_{1}=302,3+0,125 X_{1}+11,25 X_{2}+7,25 X_{1} X_{2}-15,46 X_{1}^{2}-18,1 X_{2}^{2}, \\
y_{2}=0,462-0,036 X_{1}+0,032 X_{2}-0,0075 X_{1} X_{2}-0,033 X_{1}^{2}-0,0154 X_{2}^{2} .
\end{gathered}
$$

where $X_{i}$ - codified factor meanings connected with natural meanings $x_{i}$ correlation:

$$
X_{1}=\frac{x_{1}-3}{2} ; \quad X_{2}=\frac{x_{2}-2}{1}
$$

The interpretation of equations (1) and (2) was carried out using the method based on calculation and analysis of orthogonal invariants of surfaces of the second order.

General equation of the surface of the second order is following:

$$
a_{11} x^{2}+a_{22} y^{2}+a_{33} z^{2}+2 a_{12} x y+2 a_{13} x z+2 a_{23} y z+2 a_{1} x+2 a_{2} y+2 a_{3} z+a_{0}=0,
$$

where $a_{0}, a_{i i}$ - coefficients of the equation of the second order; $x, \mathrm{y}$ and $\mathrm{z}$-differentials, which correspond to the factors $X_{1}, X_{2}$, and to response functions $y_{1}$ or $y_{2}$.

Calculated coefficients $a_{0}$ and $a_{i i}$ are demonstrated in the table 1.

Table 1. Coefficient meanings of the general equation of the surface of the second order.

\begin{tabular}{|c|c|c|c|c|c|c|c|c|c|c|}
\hline \multirow{2}{*}{$\begin{array}{c}\text { Response } \\
\text { function }\end{array}$} & \multicolumn{10}{|c|}{ Coefficient meanings } \\
\cline { 2 - 12 } & $\boldsymbol{a}_{11}$ & $\boldsymbol{a}_{22}$ & $\boldsymbol{a}_{33}$ & $\boldsymbol{a}_{12}$ & $\boldsymbol{a}_{13}$ & $\boldsymbol{a}_{23}$ & $\boldsymbol{a}_{\boldsymbol{1}}$ & $\boldsymbol{a}_{2}$ & $\boldsymbol{a}_{3}$ & $\boldsymbol{a}_{\boldsymbol{0}}$ \\
\hline$y_{1}$ & $-11,64$ & $-18,1$ & 0 & 3,625 & 0 & 0 & 0,063 & 5,635 & $-0,5$ & 302,3 \\
\hline$y_{2}$ & $-0,033$ & $-0,015$ & 0 & $-0,004$ & 0 & 0 & $-0,018$ & 0,016 & $-0,5$ & 0,462 \\
\hline
\end{tabular}

The information about configuration of surfaces of the second order which are described with the equations (1) and (2) were obtained on the basis of two invariants $I_{3}$ and $I_{4}$ composed of coefficients of the general equation. They look as following:

$$
\begin{aligned}
I_{3} & =\left|\begin{array}{lll}
a_{11} & a_{12} & a_{13} \\
a_{21} & a_{22} & a_{23} \\
a_{31} & a_{32} & a_{33}
\end{array}\right| ; \\
I_{4} & =\left|\begin{array}{llll}
a_{11} & a_{12} & a_{13} & a_{1} \\
a_{21} & a_{22} & a_{23} & a_{2} \\
a_{31} & a_{32} & a_{33} & a_{3} \\
a_{1} & a_{2} & a_{3} & a_{0}
\end{array}\right| .
\end{aligned}
$$


The graphic interpretation of the equations (2) and (3) is demonstrated in fig. 1, 2. Twodimensional cross section of the experiment sphere constitutes aa circle which radius is equal to the length the «star shoulder» and its centre coincides with the centre of the experiment.

As a result of the analysis of the data obtained there were preliminary determined the areas of factor space with optimal (maximal) numbers $y_{1}$ and $y_{2}$. The highest number of the unit value $y_{1}{ }^{\max }=304,18 \mathrm{sm}^{3} / 100 \mathrm{~g}$ was registered in the following conditions: $X_{1}{ }^{*}=0,11$ and $X_{2}{ }^{*}=0,33$, of the shape stability $-y_{2}{ }^{\max }=0,49-$ with $X_{1}{ }^{*}=0,68$ and $X_{2}{ }^{*}=1,2$, which corresponded to different points of the factor space. It is obvious that in this case it is impossible to establish optimal numbers of differentials $X_{1}$ and $X_{2}$, which would provide maximum values to $y_{1}$ and $y_{2}$ at the same time.

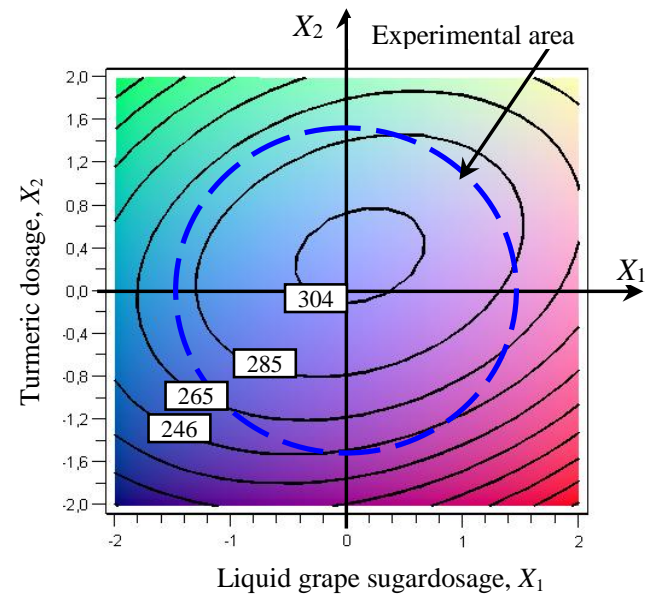

Fig. 1. Two-dimensional cross sections of the response surface $y_{1}$ (numbers represent bread unit volumey, $\left.\mathrm{sm}^{3} / 100 \mathrm{~g}\right)$

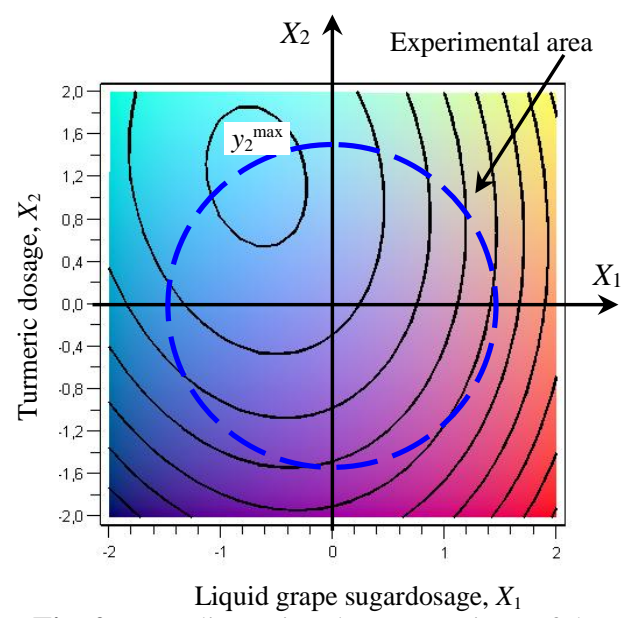

Fig. 2. Two-dimensional cross sections of the response surface $y_{2}$ (numbers represent shape stability of bread, conv. units)

The task of the optimisation in this case is multucriteria with a conflict between optimal numbers of the differentials $X_{1}, X_{2}$ and with these numbers which corresponds to every private parameter of $y_{1}, y_{2}$. It was solved using generalised additive criterion $F$, calculated basing on the method of folding private criteria:

$$
F=\sum_{i=1}^{n} \alpha_{i} \tilde{y}_{i}=\alpha_{1} \tilde{y}_{1}+\alpha_{2} \tilde{y}_{2}
$$

where $\alpha_{1}, \alpha_{2}$ - weight coefficients of relative meaning of private criteria $y_{1}$ and $y_{2} ; \tilde{y}_{1}, \tilde{y}_{2}-$ normalised private criteria.

Basing on coefficients numbers of relative spread of private criteria there were determined coefficients $\alpha_{1}$ and $\alpha_{2}$ [10]. Their calculated numbers are represented in the table 2. 
Table 2. Calculation of weight coefficients.

\begin{tabular}{|c|c|c|c|c|}
\hline $\begin{array}{c}\text { Private } \\
\text { criterion } \\
\boldsymbol{y}_{\boldsymbol{i}}\end{array}$ & $\begin{array}{c}\text { Maximal number } \\
\text { of private criterion } \\
\boldsymbol{y}_{\boldsymbol{I}} \text { max }\end{array}$ & $\begin{array}{c}\text { Minimal number of } \\
\text { private criterion } \boldsymbol{y}_{\boldsymbol{I}}^{\text {min }}\end{array}$ & $\begin{array}{c}\text { Coefficient of } \\
\text { relative spread } \boldsymbol{\delta}_{\boldsymbol{i}}\end{array}$ & $\begin{array}{c}\text { Weight } \\
\text { coefficient } \boldsymbol{\alpha}_{\boldsymbol{i}}\end{array}$ \\
\hline$y_{1}$ & 304,2 & 247,97 & 1,82 & 0,52 \\
\hline$y_{2}$ & 0,49 & 0,34 & 1,67 & 0,48 \\
\hline
\end{tabular}

Private criteria $y_{1}$ and $y_{2}$ led tonon-dimensional type of $\tilde{y}_{1}$, and $\tilde{y}_{2}$, according to the ratio $\tilde{y}_{1}=\frac{y_{i}}{y_{i}^{\text {max }}}$. The equations (1) and (2) as a result of normalisation took on new forms:

$$
\begin{gathered}
\tilde{y}_{1}=0,993+4,1 \cdot 10^{-4} X_{1}+0,037 X_{2}+0,0238 X_{1} X_{2}-0,038 X_{1}^{2}-0,059 X_{2}^{2}, \\
\tilde{y}_{2}=0,94-0,073 X_{1}+0,065 X_{2}-0,015 X_{1} X_{2}-0,067 X_{1}^{2}-0,031 X_{2}^{2} .
\end{gathered}
$$

As a result, the generalized optimisation criterion $F$, as a weighted average sum of normalised partial criteria can be determined on the basis of the equation:

$$
F=0,996-0,035 X_{1}+0,05 X_{2}+0,0048 X_{1} X_{2}-0,051 X_{1}^{2}-0,046 X_{2}^{2} \text {. }
$$

The condition for the extremum of the function (10) is the equality of partial derivatives $F\left(X_{1}, X_{2}\right)$ to zero on independent variables:

$$
\left\{\begin{array}{l}
\frac{\partial F}{\partial X_{1}}=-0,035-0,102 X_{1}+0,0048 X_{2}=0 \\
\frac{\partial F}{\partial X_{2}}=0,05+0,0048 X_{1}-0,092 X_{2}=0
\end{array} .\right.
$$

Having solved the system (11) there were determines the coordinates of the stationary point of the factor space $X_{1}{ }^{*}=-0,32$ and $X_{2}{ }^{*}=0,53$ which meet the condition of the extremum of function (10).

The verification of the found extremum was carried out using determinant which elements were represented by partial derivatives:

$$
\Delta=\left|\begin{array}{cc}
\frac{\partial^{2} F}{\partial X_{1}^{2}} & \frac{\partial^{2} F}{\partial X_{1} \partial X_{2}} \\
\frac{\partial^{2} F}{\partial X_{2} \partial X_{1}} & \frac{\partial^{2} F}{\partial X_{2}^{2}}
\end{array}\right| .
$$

There were determined partial derivatives and a number of the determinant: $\frac{\delta^{2} F}{\delta X_{1}^{2}}=-0,102$,

$$
\frac{\partial^{2} F}{\partial X_{2}^{2}}=-0,092 \frac{\partial^{2} F}{\partial X_{1} \partial X_{2}}=\frac{\partial^{2} F}{\partial X_{2} \partial X_{1}}=0,0048 \quad \Delta=\frac{\partial^{2} F}{\partial X_{1}^{2}} \cdot \frac{\partial^{2} F}{\partial X_{2}^{2}}-\left(\frac{\partial^{2} F}{\partial X_{1} \partial X_{2}}\right)^{2}=0,0094
$$

\begin{tabular}{|c|c|c|c|c|c|c|}
\hline \multicolumn{2}{|c|}{$\begin{array}{c}\text { Codified factor } \\
\text { numbers }\end{array}$} & \multicolumn{2}{|c|}{$\begin{array}{c}\text { Natural factor } \\
\text { numbers }\end{array}$} & \multirow{2}{*}{$\begin{array}{c}\text { Generalised } \\
\text { optimisation } \\
\text { criterion } F^{*}\end{array}$} & \multirow{2}{*}{$\begin{array}{c}\text { Bread unit } \\
\text { volume } y_{1}{ }^{*} \\
\text { sm }^{3} / 100 \mathrm{~g}\end{array}$} & \multirow{2}{*}{$\begin{array}{c}\text { Shape } \\
\text { stability of } \\
\text { bread } y_{1}{ }^{*}, \\
\text { conv. units }\end{array}$} \\
\hline$X_{I}^{*}$ & $X_{2}{ }^{*}$ & $x_{1}{ }^{*}, \%$ & $x_{2}{ }^{*}, \%$ & & & \\
\hline$-0,32$ & 0,53 & 2,36 & 2,53 & 0,984 & 300,72 & 0,48 \\
\hline
\end{tabular}

As in the stationary point $\Delta>0$ and $\frac{\delta^{2} F}{\delta X_{1}^{2}}<0$, it means that the point with coordinates $X_{1}{ }^{*}=-0,32$ and $X_{2}{ }^{*}=0,53$ provides a maximal number of the generalized optimisation criterion $F(10) F^{*}=0,984$. The results of the optimisation are demonstrated in the table 3 .

Table 3. Optimal parameters 
As a result of a series of parallel studies there was confined the choice of optimal factor numbers. Root-sum-square uncertainty did not exceed $0,67 \%$ which proves a sufficient correspondence of the results.

The study formed the basis for the elaboration of the technology of the bread «Merita» (TU 9110-515-02068108-2019) which is characterised by improved quality indicators, reduced energetic value and higher nutritional value on the account of additional biologically active nutrients.

This technology was applied during the test baking in the industrial conditions which allowed to register the increase of the bread output of $3 \%$ and the reduction of the technological cycle of 1,5 hour.

\section{Conclusions}

In conclusion, the use of untraditional raw materials in optimal dosages helps to increase the effectiveness of the bakery production due to the following aspects:

- bread quality improvement;

- intensification of the process of production of semi-processed goods;

- nutritional value improvement;

- increased bread outcome.

\section{Literature}

1. L.P. Bessonova, A.A. Shevcov, I.V. Mazhulina, T.N. Tertychnaya, Khleboproducty, 2, 36 (2014)

2. N.A. Berezina, A.V. Artyomov, E.V. Hmelyova, I.A. Nikitin, Khleboproducty, 9, 60 (2019)

3. K.H. Cox, A. Pipingas, A.B. Scholey, Journal of psychopharmacology, Vol. 29 (5), 642 (2015)

4. A.S. Balakina, I.V. Aksenov, N.V. Trusov, G.V. Guseva, L.I. Avrent'eva, L.V. Kravchenko, V.A. Tutel'yan, Problems of nutrition, 2, 14 (2017)

5. E.I. Ponomareva, S.I. Lukina, A.V. Odincova, E.V. Zubkova, Khleboproducty, 8, 40 (2016)

6. E.I. Ponomareva, S.I. Lukina, N.N. Alekhina, T.N. Malyutina, Workshop on industry technology (bakery products technology), Lan', 316 (2017)

7. A.G. Korotchenko, E.A. Kumagina, V.M. Smoryakova, Introduction to multi-criteria optimization, N.Novgorod, 55 (2014)

8. A.A. Zhuravlev, S.I. Lukina, E.I. Ponomareva, K.E. Roslyakova, Foods and Raw Materials. 1, 73 (2017)

9. N.N.Shchipkova, A.R. Rustanov, S.V. Kharitonova, Analytical geometry. Secondordersurfaces: trainingmanual, Orenburg: OSU, 134 (2013)

10. A.A. Zhuravlev, S.I. Lukina, E.I. Ponomareva, IV International Scientific and Technical Conference, Voronezh: VSUET, 689 (2017) 